\title{
Robert Escarpit, de la littérature à la communication
}

Hugues Hotier

\section{(2) OpenEdition \\ 12 Journals}

Édition électronique

URL : http://journals.openedition.org/communicationorganisation/1750

DOI : 10.4000/communicationorganisation. 1750

ISSN : $1775-3546$

Éditeur

Presses universitaires de Bordeaux

Édition imprimée

Date de publication : 1 novembre 1994

ISSN : 1168-5549

\section{Référence électronique}

Hugues Hotier, «Robert Escarpit, de la littérature à la communication », Communication et organisation [En ligne], 6 | 1994, mis en ligne le 26 mars 2012, consulté le 30 avril 2019. URL : http://

journals.openedition.org/communicationorganisation/1750; DOI : 10.4000/

communicationorganisation. 1750

Ce document a été généré automatiquement le 30 avril 2019.

(c) Presses universitaires de Bordeaux 


\title{
Robert Escarpit, de la littérature à la communication
}

\author{
Hugues Hotier
}

1 Quand on évoque la ville de Bordeaux, il est des personnages qui viennent immédiatement d l'esprit. Certains appartiennent d l'histoire: Alienor d'Aquitaine, mère de Richard Coeur de Lion, $d$ qui nous devons quatre siècles d'occupation anglaise, Montaigne ou Montesquieu... plus proches de nous, certains sont liés à l'actualité politique et sociale.

2 Universitaire mondialement connu - plusieurs de ses ouvrages ont été traduits dans une vingtaine de langues - homme politique régional exhibant son marxisme comme d'autres l'ostensoir, Robert Escarpit est un empêcheur de penser en rond. Alors qu'il évolue dans des milieux volontiers conservateurs, celui de l'Université comme celui de la ville, il provoque et dérange par ses idées et sa personnalité innovante. C'est précisément cette pulsion novatrice qui l'habite qui a permis d la communication de devenir une discipline d part entière et $d$ la recherche française de n'être pas en retard. Le débat Escarpit-Mac Luhan a bien eu lieu et reconnaissons que nous n'en étions pas peu fiers.

Communication \& Organisation a rendu visite au père des sciences de l'Information et de la communication.

4 En Europe, le mot communication est lié au nom d'un homme qui en a fait une discipline universitaire et a contribué à la définir par une approche théorique ${ }^{1}$ dont chacun reconnait à la fois le mérite et la qualité. Auteur de 68 ouvrages, dont une vingtaine de romans, Robert Escarpit a été longtemps professeur de littérature comparée à l'Université de Bordeaux 3 où il a fondé la première école universitaire française de communication.

5 En 1948, alors qu'il exerce au Mexique, il publie, en espagnol, un traité de la littérature française qui comporte un chapitre intitulé «Les trois dimensions de la littérature » dans lequel il prend en compte le lecteur comme destinataire et récepteur d'un message, le texte, émis par l'auteur. L'approche est pour le moins nouvelle et laisse entrevoir les développements ultérieurs qui sortiront du domaine littéraire. 
6 Vers 1957, avec la maturité que confère la quarantaine, commence à lui peser « ce métier qui consiste à produire des discours sur les discours des autres ». Avec le recul, il reconnaît la l'influence d'Henri Peyre et de Thibaudet-Château, professeur de psychologie à l'Université de Bordeaux. C'est d'ailleurs lui qui lui conseille de lire les travaux de Lehmann, lequel a établi l'âge de la meilleure performance dans à peu près tous les domaines de l'activité humaine. À peu près, puisqu'échappe notamment à cette savante étude le domaine de la littérature. Ce sera le sujet d'un ouvrage de la collection « Que saisje ?» traduit en vingt-trois langues, le chinois compris, qui est toujours d'actualité et que des générations d'étudiants et de chercheurs ont lus : Sociologie de la littérature.

7 Paradoxe, Robert Escarpit n'avait jamais étudié la sociologie et c'est le succès de cette approche sociologique de la littérature qui lui mettra le pied à l'étrier pour sa démarche en communication. D'abord, parce que le succès de son livre lui vaut une certaine reconnaissance dans les milieux universitaires. On l'autorise à fonder un centre de recherche et même on lui nomme une secrétaire et une assistante. Ensuite, parce que ce succès lui permet de rencontrer d'autres chercheurs qui compteront pour lui. Parmi ceux-ci, Alphonse Silberman, professeur à l'Université de Cologne, le mathématicien français Elie Roubine et Julien Behrstok, chef du Département du Livre à l'UNESCO qui lui confie de nombreuses missions et lui fait part d'un de ses rêves: créer dans cette institution internationale... un département de communication.

8 Sous l'égide de l'UNESCO, il publie La révolution du livre, ouvrage dans lequel il pose une question qui deviendra le titre d'un article pour la revue yougoslave Philoski Pregled: l'acte littéraire est-il un acte de communication? Nous sommes en 1962, Robert Escarpit vient de franchir un pas décisif qui le mènera du domaine particulier de la littérature au champ plus large de la communication. Il fonde alors, à Bordeaux, le Centre de Sociologie des Faits littéraires - et non "de la littérature" car il ne faut pas effrayer le monde universitaire- qui deviendra successivement l'Institut de la Littérature et des Techniques Artistiques de Masse, en 1965, puis le Laboratoire Associé des Sciences de l'Information et de la Communication (LASIC), reconnu, au même titre que l'ILTAM, par le CNRS.

9 Mais cet homme d'action ne se limite pas à la recherche. Ayant publié ses premiers articles à l'âge de seize ans, Robert Escarpit s'est toujours et avant tout senti journaliste. Il entrera au Monde dès sa fondation en 1944 et sera internationalement connu par les célèbres billets qu'il y écrira à partir de 1949. En trente ans, il en publiera plus de neuf mille!

10 Un jour de 1967, dans son billet quotidien, il raille Christian Fouchet dont il a peut-être oublié qu'il est aussi son ministre de tutelle, le ministre de l'Éducation Nationale à laquelle il appartient toujours. Le ministre le convoque et, après les reproches prévus, lui demande :

11 « - Et cette communication dont vous parlez tant, à quoi cela sert-il ?

12 - C'est utile. Cela pourrait vous servir à vous-même. Je vous ai observé à la télévision. Vous avez un tic: vous faites non de la tête à chaque fois que vous dîtes oui...vous êtes libre à déjeuner? " Demanda, une fois la surprise passée, ce ministre qui avait plus de curiosité que d'humour.

13 Et c'est ainsi qu'a table, Robert Escarpit et Christian Fouchet créèrent le premier IUT ${ }^{2}$ de communication. Il rentre à Bordeaux avec cette bonne nouvelle et des moyens pour lui donner corps : quatre postes d'enseignants et un budget d'équipement de 290000 francs. 

recherche, elle devient matière à enseigner. Il faut dire que, quelques années plus tôt, il avait profité d'une opportunité pour créer une licence professionnelle, la licence de journalisme, avec l'aide de deux journalistes connus : Albert Rêche et Henri Amouroux. Avec sa licence de journalisme et son IUT carrières de l'information, l'Université Bordeaux 3 devenait un pôle de formation aux métiers de l'information et de la communication ${ }^{3}$.

Il restait à faire reconnaitre la discipline par l'Université, avec un U majuscule, car en France rien n'existe tant que les textes juridiques ou réglementaires ne l'ont pas officiellement signifié... Il fallait pour cette troisième étape "débaucher » quelques universitaires appartenant à d'autres sections du Comité Consultatif des Universités. Le $\mathrm{CCU}$, qui changea plusieurs fois de nom et de sigle depuis, est l'organisme officiel qui régit les carrières des universitaires. Tous les enseignants de statut universitaire sont répartis en sections correspondant à des disciplines. Pour revendiquer la création d'une nouvelle section, ce qui équivaut à faire reconnaitre un nouveau champ disciplinaire, la première condition est qu'au moins seize professeurs et maîtres de conférences (à l'époque «maittres-assistants ») en manifestent le désir. Il en trouve, et non des moindres : le célèbre sémioticien Roland Barthe, le spécialiste des sciences et techniques de la documentation Jean Meyriat, le linguiste Bernard Quémada, l'historien André-Jean Tudesq sont parmi les premiers à quitter leur section pour constituer « la $52^{\mathrm{e}}$ section du CCU » que, par souci d'œcuménisme, on dénommera «sciences de l'information et de la communication ». Sciences s'écrit avec un s et l'information et la communication sont mises sur un pied d'égalité. Le monde professionnel soutiendra la démarche et la aussi on retrouvera des personnalités tel Marcel Bleustein-Blanchet, homme de communication s'il en fut. Robert Escarpit se flatte d'être ainsi devenu, en 1974, le premier professeur français de sciences de l'information et de la communication. Et quand, en 1975, il m'envoya, comme à d'autres, une lettre m'invitant à rejoindre ceux qui avaient créé cette nouvelle section je n'hésitai pas un instant ${ }^{4}$. Je travaillais alors sur la presse à sensation, que je traitais d'un point de vue linguistique et la linguistique était devenue une des sciences qui pouvaient s'appliquer à la presse. Nous nous trouvâmes alors de plain pied dans les sciences de l'information et de la communication. Et même le mot information, polysémique jusqu'à l'ambiguïté, devenait acceptable par tous.

Robert Escarpit n'en est pas pour autant imprécis quand on lui demande des définitions. Pour lui les choses sont claires :

«La communication est le véhicule de l'information. J'appelle information tout message qui perturbe l'ordre établi. Mais pour qu'il y ait communication, il faut un stimulus et une réponse. Ce n'est pas quand je dis «allo? » que la communication est établie, c'est quand l'autre a répondu « allo ». C'est l'autre qui fait le message. »

On affuble du nom de communication ce qui n'en est pas. Ainsi, dans la publicité, la réponse est binaire: j'achète ou je n'achète pas. Face à la télévision, la réponse est binaire: je zappe ou je ne zappe pas. On ne peut pas appe1er communication l'acte d'acheter le produit ou l'acte d'éteindre le téléviseur. Pour qu'il y ait communication, il faut des réponses riches. C'est la réponse qui fait la communication comme c'est le récepteur qui fait le sens.

La communication est aussi dépendante du milieu dans lequel elle s'exerce. Plus il y a de monde, moins il y a de communication. Dans un groupe d'une douzaine de personnes, il peut y avoir communication. Mais ce qu'on appelle « communication de masse » n'existe

Communication et organisation, 6 | 2012 
pas. La notion de communication implique une richesse de réponses et une vraie interactivité. Dans la communication, la notion de relation est une notion essentielle.

Il faudrait encore prendre en compte l'établissement de cette relation. Ainsi, quand Michel Touret, bordelais d'origine, annonce la météo à la télévision, il ne manque jamais de dire la température qu'il fera à Bordeaux. Ceux qui savent qu'il est bordelais, comprennent le clin d'œil et cela crée une relation. Dans mon village des Pyrénées quand le maire s'adresse à un de ses amis du village, il s'arrête à deux mètres de lui et lui parle en béarnais. Quand il agit en tant que maire adressant la parole à son administré, il s'arrête à un mètre cinquante et parle français. Et l'attitude aussi bien que la langue donnent des indications sur la nature de la relation qui s'établira. »

Quand il parle de ses recherches, Robert Escarpit n'emploie pas le mot travail. II lui semblerait inconvenant de parler ainsi pour désigner son plaisir. S'il regrette qu'il y ait trop de descriptions des applications et pas assez de réflexion théorique en matière de communication, s'il déplore que trop de gens déclarent «faire de la communication », il semble qu'il ait abandonné le désir de théoriser. Après tout, il a déjà beaucoup donné en la matière. Et il a bien le droit de s'amuser à dépouiller le Who's who pour définir « le notable fin de siècle ». Robert Escarpit n'a pas fini de nous étonner !

\section{NOTES}

1. ESCARPIT Robert Théorie générale de l'information et de la communication Paris, Hachette, 1976, (2e édition 1993), Collection Hachette Université

2. Institut Universitaire de Technologie, établissements dérogatoires de premier cycle qui préparent en deux ans è des carrières professionnelles

3. Cette licence de journalisme sera la base de l'Unité Pluridisciplinaire des Techniques d'Expression et de Communication (UPTEC), fondée par Robert Escarpit en 1969 et qui prendra, en 1980, le nom de Institut des Sciences de l'information et de la Communication (ISIC)

4. Je ne savais pas alors que moins de dix ans plus tard je succèderais à Robert Escarpit à l'Université de Bordeaux. 